\title{
Dynamic evidential networks in system reliability analysis: A Dempster Shafer Approach
}

\author{
Philippe WEBER and Christophe SIMON
}

\begin{abstract}
Nowadays, complex manufacturing processes have to be dynamically modeled to estimate their reliability. Moreover the results computed with classical methods need to be reinforced by managing the uncertainty. To address these difficulties, this paper presents a new method for modeling and analyzing the system reliability based on Dynamic Evidential Networks (DEN). This method allows modeling the influence of time and uncertainty on the failure and degradation of the system. The DEN graphical structure provides an easy way to specify the dependencies and, hence, to provide a compact representation of the system based on the Dempster Shafer theory. In addition, the DEN formalism is associated to simulation tools that enable an efficient processing for the models. A small system is used to compare the reliability estimations obtained by the proposed DEN model and those obtained by the classical Markov Chain.
\end{abstract}

\section{INTRODUCTION}

One of the main challenges of the Extended Entreprise is to maintain and optimize dynamically the quality of the services delivered by industrial objects along their life cycle. The goal is to design decision-making aid systems to maintain the process in operation. Nevertheless, a decision is taken without a perfect perception of all the system states. This partial perception argues in favor of using a probabilistic estimation of the system state. Nevertheless, several alternatives mathematical representations of both stochastic and epistemic uncertainties have been proposed, including evidence theory, possibility theory and fuzzy set theory [1], [2]. Evidence theory is a promising alternative allowing a fuller representation of the implications of the knowledge uncertainty than in the probabilistic representation [3]. Works on system safety and Evidential Networks (EN) have been developed recently [4]. The authors used the exact inference algorithm proposed by Jensen [5] to compute the marginal mass distributions in the network.

The purpose of this paper is to introduce Dynamic Evidential Networks (DEN) in a way similar to the Markov Chains (MC) [6] to deals with epistemic uncertainty. The considered problems are those involving systems whose dynamics can be modeled as stochastic processes. The current system state determine the probability distribution over the next states. In the work reported here a study dedicated to the comparison between MC and DEN for system reliability estimation is proposed.

Section 2 presents the limits of MC model when epistemic uncertainty is present in the knowledge. Section 3 presents the Dempster Shafer theory and the new Dynamic Evidential Network (DEN) formalism. The proposed methodology is an original formalization of a system reliability model by DEN proposed in section 4. A simulation of a classical system is developed in section 4 to compare MC and DEN models. Finally, section 5 presents conclusion and perspectives.

\section{Problem Statement}

To take the uncertainty into account, it is possible to consider the process state as a random variable that takes its values from a finite state space corresponding to the possible process states. A MC allows modeling the dynamics of sequences taken by these states [7]. The uncertainty taken into account by $\mathrm{MC}$ is the stochastic one. But, as databases of reliability are used to define the failure rates of the components [8]-[11], and the values of failure rates are associated to several classes of components, some databases [8], [12], [13] supply the lower and upper values, mean or median values or error factors. Besides, Kletz [14] pointed out that the parameter in reliability may change with a factor 3 to 10. Some authors [15], [16] recommend to introduce a correction coefficient to modify the failure rate of the databases according to the operational conditions of the component. This imprecision in the knowledge of failure rates induces an epistemic uncertainty which is not suitably managed by stochastic models like MC.

When upper and lower bounds of the failure rates $\left(\underline{\lambda}_{X}, \bar{\lambda}_{X}\right)$ of a component $X$ are given, it is easy to define the bounds of the component reliability at mission time $T_{i}$ :

$$
\left[\underline{P}_{X}, \bar{P}_{X}\right]=1-\exp \left(\left[\underline{\lambda}_{X}, \bar{\lambda}_{X}\right] \cdot T_{i}\right)
$$

Where $\underline{P}_{X}$ is the lower probability of the hypothesis $(X=$ $\{U p\})$ and $\bar{P}_{X}$ the corresponding upper probability.

In the following, a method based on Dempster Shafer theory to manage the epistemic uncertainty is presented to model system reliability. It is based on Dynamic Evidential Networks.

\section{EVIDENTIAL NETWORK THEORY}

The Evidential Network model is based on graph theory and Dempster Shafer theory. Each node represents a variable and arcs indicate direct conditional relations between the connected nodes. The Dynamic Evidential Network (DEN) takes into account the time by defining different nodes to model variables with respect to different time slices. 


\section{A. Evidence theory}

In the Dempster-Shafer theory, let us consider the set of $r$ mutual exclusive hypothesis called the frame of discernment:

$$
\Omega=\left\{H_{1}, H_{2}, \ldots H_{r}\right\}
$$

The basic belief assignment follows the function:

$$
m: 2^{\Omega} \rightarrow[0,1]
$$

with

$$
\sum_{A_{i} \in 2^{\Omega}} m\left(A_{i}\right)=1
$$

and

$$
2^{\Omega}=\left\{\left\{H_{1}\right\}, \ldots,\left\{H_{r}\right\},\left\{H_{1}, H_{2}\right\}, \ldots,\left\{H_{1} \ldots H_{r}\right\},\{\emptyset\}\right\}
$$

Each $A_{i} \in 2^{\Omega}$ with $m\left(A_{i}\right)>0$ is call a focal element. The constraint $m(\emptyset)=0$ is not necessary but this condition supposes that all hypotheses are known. Therefore $\Omega$ is assumed to be exhaustive [17]. The epistemic uncertainty is modeled by focal elements describing ambiguity between several hypotheses. For instance, $A_{i}=\left\{H_{1}, H_{2}\right\}$ denotes that the hypothesis $H_{1}$ or $H_{2}$ is respected but it is not known which one exactly.

\section{B. Plausibility and belief functions}

The belief mass distribution is used to compute the upper and lower bounds of probability. This interval contains the probability of a set of hypotheses (focal elements) and, is limited by two non-additive measures: the belief $(\mathrm{Bel})$ and plausibility $(P l s)$ of the focal element [18].

The measure of the belief $\operatorname{Bel}\left(A_{i}\right)$ in the focal element $A_{i}$ can be interpreted as the total amount of justified support given to $A_{i}$. It defines the lower bound of probability that the focal element exists. It is computed by the sum of all belief masses of a subset of hypotheses $B$ that contribute to the existence of $A_{i}$ with $B$ included in $A_{i}$.

$$
P l s\left(A_{i}\right)=\sum_{B \mid A_{i} \cap B \neq \emptyset} m(B)
$$

The measure of plausibility $P l s\left(A_{i}\right)$ quantifies the maximum amount of specific support that could be given to $A_{i}$, if justified by additional information. This upper limit is computed by the sum of all the belief masses of the subsets of hypotheses $B$ such that the intersection between $B$ and $A_{i}$ is not empty.

$$
\operatorname{Bel}\left(A_{i}\right)=\sum_{B \mid B \subseteq A_{i}} m(B)
$$

As Dempster Shafer Theory defines capacities of order $\infty$ and it verifies the assumptions of regular monotone measures (see [2]), the following property is given:

$$
\operatorname{Bel}\left(A_{i}\right) \leq P\left(A_{i}\right) \leq P l s\left(A_{i}\right)
$$

The property eq.6 is well known, Shafer has already defined it in his works in 1976 [19].

\section{The Evidential Network}

Evidential Networks are directed acyclic graphs used to represent uncertain knowledge in Artificial Intelligence [4]. A EN is defined as a couple: $G=((N, A), M)$, where $(N, A)$ represents the graph; $N$ is a set of nodes; $A$ is a set of arcs; $M$ represents the set of conditional mass distributions that quantify dependencies between the variables.

A discrete random variable is represented by a node $X \in N$ with a finite number of focal elements. The focal set of $X$ is defined by $S_{X}=\left\{A_{1}^{X}, A_{2}^{X} \ldots A_{i}^{X} \ldots\right\}$ with $A_{i}^{X} \in 2^{\Omega *}$ and $\Omega *=\left\{H_{1}^{X}, \ldots, H_{r}^{X}\right\}$. The vector $m(X)$ denotes a belief mass distribution over the focal set $S_{X}$, where $m\left(A_{i}^{X}\right)$ is the belief mass assignment to the focal element $A_{i}^{X}$. In the graph depicted in figure 1, the nodes $X$ and $Y$ are linked by an arc. If $(X, Y) \in A$ and $(Y, X) \notin A$ then $X$ is considered as a parent of $Y$. The parent set of a node $Y$ is defined as $p a(Y)=X$.

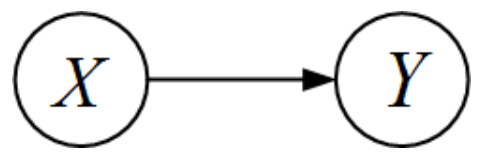

Fig. 1. A basic Evidential Network.

The set $M$ represents the Conditional Mass distribution Tables (CMT). Then, each node has an associated CMT. For instance, in figure 1 , the nodes $X$ and $Y$ are defined over the focal sets $S_{X}:\left\{A_{1}^{X}, \ldots, A_{Q}^{X}\right\}$ and $S_{Y}:\left\{A_{1}^{Y}, \ldots, A_{P}^{Y}\right\}$. Then, the CMT of $Y$ is defined by the conditional belief mass table $m(Y \mid X)$ over each $Y$ focal elements knowing the focal elements of its parent $X$. This CMT is defined as a matrix:

$$
M(Y \mid p a(Y))=\left[\begin{array}{lll}
m\left(A_{1}^{Y} \mid A_{1}^{X}\right) & \cdots & m\left(A_{P}^{Y} \mid A_{1}^{X}\right) \\
\vdots & & \vdots \\
m\left(A_{1}^{Y} \mid A_{Q}^{X}\right) & \cdots & m\left(A_{P}^{Y} \mid A_{Q}^{X}\right)
\end{array}\right]
$$

Concerning the root nodes, i.e. the node without parents, the CMT contains only one row describing the a priori mass distribution of each focal element.

Various inference algorithms can be used to compute marginal mass distributions in the Evidential Network. The most classical one relies on the use of a junction tree. This algorithm is used in Bayesian Networks and more explications can be found in [5, pp.76]. Inference in EN allows taking into account any focal element of a variable observation (an event or knowledge) for the updating of the mass distribution of each variable.

Inference computes node mass distributions knowing the focal set of one or several variables. Without any event observation, the computation is based on the a priori mass distribution of the root nodes. When observations are made, the knowledge is integrated in the network and the mass distributions over the variables focal elements are updated.

Knowledge is formalised as evidence. A hard evidence of the random variable $X$ indicates that the mass assigned to its focal elements is concentred only to one focal element of 
$S_{X}:\left\{A_{1}^{X}, \ldots, A_{Q}^{X}\right\}$. For instance $m\left(A_{1}^{X}\right)=1$ and $m\left(A_{q \neq 1}^{X}\right)=0$. Nevertheless, when this knowledge is uncertain, soft evidence can be used (see [20]). A soft evidence for a node $X$ is defined as any evidence that enables to update the prior mass distribution values for the focal elements of $X$. For example, the belief mass is assigned to the focal element $A_{1}^{X}$ and $A_{Q}^{X}$ with the same mass and not in the other focal elements: $m\left(A_{1}^{X}\right)=0.5, m\left(A_{Q}^{X}\right)=0.5$ and $m\left(A_{q \neq\{1, Q\}}^{X}\right)=0$.

To compute belief and plausibility measures, we use equations 5 and 4 . As these measures are non additives, they cannot be computed in one node if the tool used verifies the additivity axioms. Simon and Weber [4] have proposed to compute these measures on a variable in the network with two separated nodes as shown in figure 2.

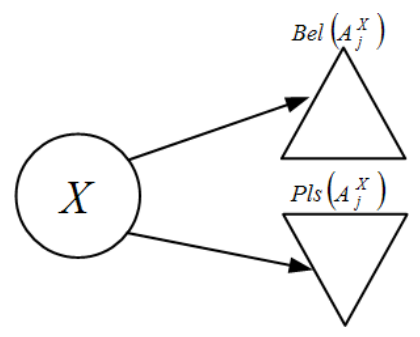

Fig. 2. Computing nodes of $\mathrm{Bel}$ and $\mathrm{Pls}$ measures

The conditional mass table of a belief node follows the table I where we introduce the doubt modality as the contrary of believe.

TABLE I

$\mathrm{CMT}$ of $\operatorname{Bel}\left(A_{j}^{X}\right)$

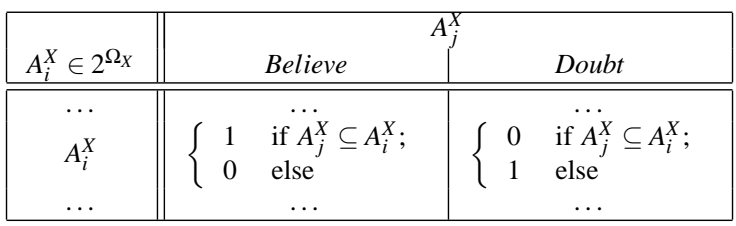

On the same way the node that computes the plausibility measure contains the conditional mass table given in table II where disbelief is introduced as the contrary of plausibility.

TABLE II

CMT of $P l s\left(H_{i}^{X}\right)$

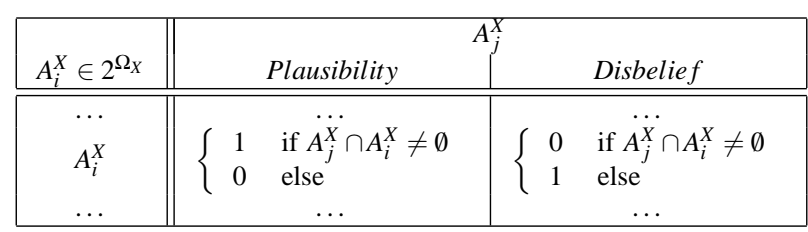

Besides, we can note that these tables can be used to compute plausibility and belief measures on each state of a component or node of the network in order to give information on probability boxes that bound the real value of the probability for components or subsystems (cf. eq.6).

\section{Dynamic Evidential Network}

A Dynamic Evidential Network is an EN including a temporal dimension. This new dimension is managed by time-indexed variables. The variable is represented at time step $k$ by a node $X_{k} \in N$ with a finite number of focal elements $S_{X_{k}}:\left\{A_{1}^{X_{k}}, \ldots, A_{Q}^{X_{k}}\right\} . m\left(A_{1}^{X_{k}}\right)$ denotes the belief mass assigned to the focal element $A_{1}^{X}$ at time step $k$. Several time stages are represented by several sets of nodes $N_{0}, \ldots, N_{k} . N_{k}$ includes all the random variables relative to the time slice $k$.

An arc linking two variables belonging to different time slices represents a temporal variation of the belief mass and models the dependence between these variables. Then, DEN allows to model random variables and their impacts on the future distribution of other variables. Defining these impacts as transition-belief masses between the focal elements of the variable at time step $k$ and those at time step $k+1$ leads to the definition of CMT relative to inter-time slices, as it is defined in eq. 7 .

$$
M\left(X_{k+1} \mid X_{k}\right)=\left[\begin{array}{lll}
m\left(A_{1}^{X_{k+1}} \mid A_{1}^{X_{k}}\right) & \cdots & m\left(A_{Q}^{X_{k+1}} \mid A_{1}^{X_{k}}\right) \\
\vdots & & \vdots \\
m\left(A_{1}^{X_{k+1}} \mid A_{Q}^{X_{k}}\right) & \cdots & m\left(A_{Q}^{X_{k+1}} \mid A_{Q}^{X_{k}}\right)
\end{array}\right]
$$

With this model, the future slice $(k+1)$ is conditionally independent of the past given the present $(k)$, which means that the CMT $M\left(X_{k+1} \mid p a\left(X_{k+1}\right)\right)$ respects properties similar to the Markov properties.

Starting from an observed situation at time step $k=0$, the belief mass distribution $m\left(X_{k}\right)$ over $n_{i}$ focal sets is computed by the DEN inference. To compute $m\left(X_{k+T}\right)$, the proposed solution keeps a compact network form. This solution is based on iterative inferences. The notion of time is introduced through inference. Indeed, it is possible to compute the belief mass distribution of any variable $X_{i}$ at time step $k+1$ based on the belief mass distribution corresponding to time step $k$. The belief mass distributions at time step $k+2 \ldots$ are computed using successive inferences. Then, a network with only two time slices is defined. The first slice contains the nodes corresponding to the current time step $(k)$, the second one those of the following time step $(k+1)$. Observations, introduced as hard evidence or belief mass distributions, are only realized in the current time slice $(k)$. The time increment is carried out by setting the computed marginal belief masses of the node at time step $k+1$ as observations for its corresponding node in the previous time slice.

Moreover, the CMT is equivalent to the Markovian model of the variable $X$ if $p a\left(X_{k+1}\right)=X_{k}$ and $S_{X_{k}}=S_{X_{k+1}}$ and without mass assignment to epistemic focal elements.

\section{DEN TO MODEL RELIABILITY}

\section{A. Evidential Network to model system reliability}

As mentioned previously, the probist reliability [21] of a system with low complexity may be modeled by a variable 


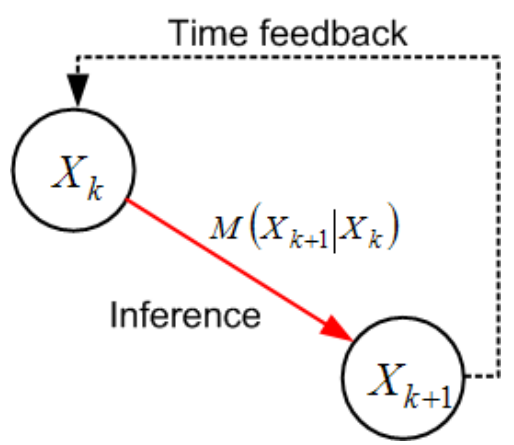

Fig. 3. A DEN for the random variable $\mathrm{X}$.

$X$ with two hypotheses. The corresponding frame of discernment is then described by:

$$
\Omega=\{U p, \text { Down }\}
$$

The set of focal elements is defined by $2^{\Omega}=$ $\{\{U p\},\{$ Down $\},\{U p$, Down $\},\{\emptyset\}\}$.

In probist reliability, the constraint $m(\emptyset)=0$ is assumed, thus the possibility to allocate a quantity to the focal set $\{U p$, Down $\}$ softens the probability framework. The role of this focal element is to characterize the ignorance on the real probability distribution over the system states without commitment. It means that the system can be in the state $\{U p\}$ or $\{$ Down $\}$. Of course, it should not be considered that the system can be in the two states simultaneously. This ignorance is an epistemic uncertainty and is described by the epistemic state $\{U p$, Down $\}$.

When $\underline{P}_{X}$ is the lower probability of the hypothesis $\{U p\}$ and $\bar{P}_{X}$ the corresponding upper probability, the upper and lower bounds of the reliability $\left(\underline{P}_{X}, \bar{P}_{X}\right)$ are easily translated in the $\mathrm{EN}$ to define the a priori belief mass assignment of $X$ :

$$
\begin{aligned}
& m(\{U p\})=\underline{P}_{X} \\
& m(\{\text { Down }\})=1-\bar{P}_{X} \\
& m(\{U p, \text { Down }\})=\bar{P}_{X}-\underline{P}_{X}
\end{aligned}
$$

Then applied the eq.4 and $5 \operatorname{Pls}(\{U p\})=\bar{P}_{X}$ and $\operatorname{Bel}(\{U p\})=\underline{P}_{X}$.

\section{B. Dynamic Evidential Network to model entities Markovian behavior}

The reliability is modeled as a DEN made of two nodes as presented in figure 3. A model of component $X$ reliability with uncertainty in failure rate is easily translated into a DEN model. Two nodes are defined to model the random variable at time slices $(k)$ and $(k+1): X_{k}$ and $X_{k+1}$ with the focal sets $\{\{U p\},\{$ Down $\},\{U p$, Down $\}\}$.

The upper and lower bounds of the failure rates $\left(\underline{\lambda}_{X}, \bar{\lambda}_{X}\right)$ of the component $X$ are used to define the Conditional belief Mass Table: $M\left(X_{k+1} \mid X_{k}\right)$. The parameters of $M\left(X_{k+1} \mid X_{k}\right)$ are defined from the failure rates $\left(\underline{\lambda}_{X}, \bar{\lambda}_{X}\right)$ (eq.11).

\section{Explanations:}

1) If the component is up then it may fall down but there is uncertainty in the failure probability. To determine the belief mass that the component is down we propose to model the uncertainty using the epistemic hypothesis $\{U p, D o w n\}$. Then the transition belief mass $m\left(\{\text { Down }\}^{k+1} \mid\{U p\}^{k}\right)=\underline{\lambda}_{X}$ corresponds to the minimum of belief that the component fall down (it is sure that the component is down after the transition time). The transition mass $m\left(\{U p, \text { Down }\}^{k+1} \mid\{U p\}^{k}\right)=$ $\bar{\lambda}_{X}-\underline{\lambda}_{X}$ corresponds to the uncertainty of the component hypothesis after the transition time. There is an ambiguity between the hypotheses $\{U p\}$ and $\{$ Down $\}$. Therefore $m\left(\{U p\}^{k+1} \mid\{U p\}^{k}\right)=1-\bar{\lambda}_{X}$ corresponds to the belief mass transition that it is sure that there is no failure. This mass is computed by:

$$
\begin{aligned}
m\left(\{U p\}^{k+1} \mid\{U p\}^{k}\right) & =1-\left(\underline{\lambda}_{X}+\bar{\lambda}_{X}-\underline{\lambda}_{X}\right) \\
& =1-\bar{\lambda}_{X}
\end{aligned}
$$

2) When the component is down, it is impossible that the component becomes up because no reparation is considered in reliability analysis. Therefore, $m\{U p\}=0, m\left(\{U p\}^{k+1} \mid\{\text { Down }\}^{k}\right)=0$ and $m\left(\{\text { Down }\}^{k+1} \mid\{\text { Down }\}^{k}\right)=1$.

3) In the epistemic uncertainty, the component is up or down. Let us consider that the component is up. There exists a transition mass modeling the failure of the component after the transition time and leading to the hypothesis $\{$ Down $\}$. This belief transition mass allocated to $\{$ Down $\}$ corresponds to the minimum of belief that the component fail down i.e. $\underline{\lambda}_{X}$. If the component is down then the component stay down. This is the reason why $m\left(\{\text { Down }\}^{k+1} \mid\{U p, \text { Down }\}^{k}\right)=\underline{\lambda}_{X}$ and $m\left(\{U p\}^{k+1} \mid\{U p, \text { Down }\}^{k}\right)=0$.

\section{EN to model independent failure of components}

A Fault Tree (FT) allows describing the logic of the propagation of failures through the system. This method allows to model the reliability of the system assuming the hypothesis of independence between the events (failures) affecting the entities. The paper [4] shows the equivalence between FT and EN in the case of uncertainty. The CMTs are used to define the OR/AND gates.

\section{Application}

The proposed method is applied to a classical example of reliability analysis. This example allows comparing easily the proposed method based on DEN with those using MC.

Figure 4 describes the system. Three valves $V_{i}$ are used to distribute a fluid. Each valve has one failure modes. The upper and lower bounds of the failure rates are given below:

$$
\begin{array}{lll}
\bar{\lambda}_{1}=2.10^{-3} & \bar{\lambda}_{2}=3.10^{-3} & \bar{\lambda}_{3}=4.10^{-3} \\
\underline{\lambda}_{1}=1.10^{-3} & \underline{\lambda}_{2}=2.10^{-3} & \underline{\lambda}_{3}=3.10^{-3}
\end{array}
$$

An equivalent model of this $\mathrm{MC}$ is realized by means of the DEN depicted in figure 6 including the uncertainty on failure rates.

Following equation 11 for temporal nodes, the temporal conditional mass table of each valves is computed. Figure 7 


$$
\begin{aligned}
M\left(X_{k+1} \mid X_{k}\right) & =\left[\begin{array}{ccc}
m\left(\{U p\}^{k+1} \mid\{U p\}^{k}\right) & m\left(\{\text { Down }\}^{k+1} \mid\{U p\}^{k}\right) & m\left(\{U p, \text { Down }\}^{k+1} \mid\{U p\}^{k}\right) \\
m\left(\{U p\}^{k+1} \mid\{\text { Down }\}^{k}\right) & m\left(\{\text { Down }\}^{k+1} \mid\{\text { Down }\}^{k}\right) & m\left(\{U p, \text { Down }\}^{k+1} \mid\{\text { Down }\}^{k}\right) \\
m\left(\{U p\}^{k+1} \mid\{U p, \text { Down }\}^{k}\right) & m\left(\{\text { Down }\}^{k+1} \mid\{U p, \text { Down }\}^{k}\right) & m\left(\{U p, \text { Down }\}^{k+1} \mid\{U p, \text { Down }\}^{k}\right)
\end{array}\right] \\
& =\left[\begin{array}{ccc}
1-\bar{\lambda}_{X} & \underline{\lambda}_{X} & \bar{\lambda}_{X}-\underline{\lambda}_{X} \\
0 & 1 & 0 \\
0 & \underline{\lambda}_{X} & 1-\underline{\lambda}_{X}
\end{array}\right]
\end{aligned}
$$

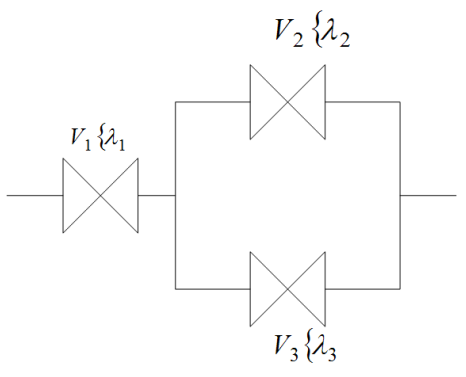

Fig. 4. Valve system.

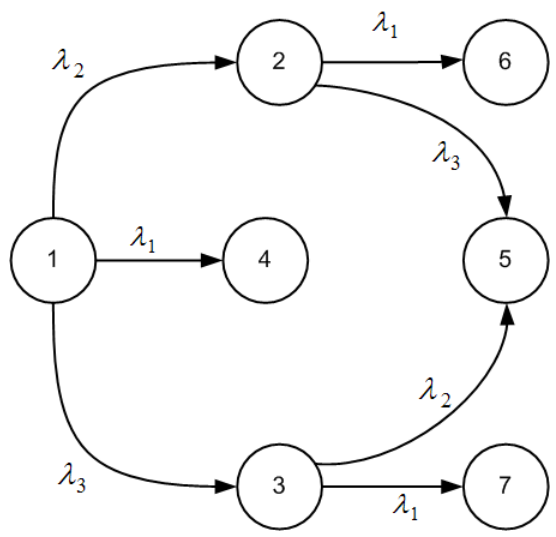

Fig. 5. Markov Chain of the system.

shows the CMT for the temporal node between $V_{1}^{k}$ and $V_{1}^{k+1}$.

The propagation through the Dynamic Evidential Network model allows taking into account the dependency between the failure modes for the computation of the system reliability $R(k)$. Thus, the node $R(k)$ computes the reliability of the system from the mass distributions over the states of valves according to the conditional mass table given in figure 8 .

Inferences are realized thanks to BayesiaLab $\left.{ }^{(}\right)$software

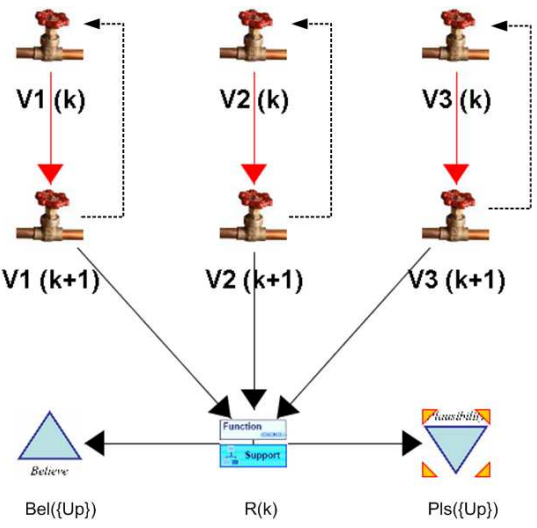

Fig. 6. DEN model.

\begin{tabular}{|c|r|r|r|}
\hline V1 $(k)$ & \multicolumn{1}{|c|}{ Up } & \multicolumn{1}{|c|}{ Down } & \multicolumn{1}{c|}{ UpDown } \\
\hline Up & 99,800 & 0,100 & 0,100 \\
\hline Down & 0,000 & 100,000 & 0,000 \\
\hline UpDown & 0,000 & 0,100 & 99,900 \\
\hline
\end{tabular}

Fig. 7. Temporal CMT of $V_{1}^{k+1}$.

that uses an iterative procedure (http://www.bayesia.com). BayesiaLab $^{\circledR}$ is used to simulate the DEN modeling $R(k)$ of the system over 1200 time steps depicted in figure 9. The results of MC are also added to this figure for comparison.

The results obtained by means of DEN are close to those obtained with two different simulations of the MC model, the first simulation with lower bound of the failure rates and the second with the upper bound of the failure rates. They are in fact more precise in DEN. Indeed, the differences are due to the approximation made assuming in the Markov model that the simultaneous failures cannot occurred, this hypothesis is not assumed in the DEN model. 


\begin{tabular}{|c|c|c|c|c|c|}
\hline V1 $(k+1)$ & V2 $(k+1)$ & V3 $(k+1)$ & Up & Down & UpDown \\
\hline \multirow{9}{*}{ Up } & \multirow{3}{*}{ Up } & Up & 100,000 & 0,000 & 0,000 \\
\hline & & Down & 100,000 & 0,000 & 0,000 \\
\hline & & UpDown & 100,000 & 0,000 & 0,000 \\
\hline & \multirow{3}{*}{ Down } & Up & 100,000 & 0,000 & 0,000 \\
\hline & & Down & 0,000 & 100,000 & 0,000 \\
\hline & & UpDown & 0,000 & 0,000 & 100,000 \\
\hline & \multirow{3}{*}{ UpDown } & Up & 100,000 & 0,000 & 0,000 \\
\hline & & Down & 0,000 & 0,000 & 100,000 \\
\hline & & UpDown & 0,000 & 0,000 & 100,000 \\
\hline \multirow{9}{*}{ Down } & \multirow{3}{*}{ Up } & Up & 0,000 & 100,000 & 0,000 \\
\hline & & Down & 0,000 & 100,000 & 0,000 \\
\hline & & UpDown & 0,000 & 100,000 & 0,000 \\
\hline & \multirow{3}{*}{ Down } & Up & 0,000 & 100,000 & 0,000 \\
\hline & & Down & 0,000 & 100,000 & 0,000 \\
\hline & & UpDown & 0,000 & 100,000 & 0,000 \\
\hline & \multirow{3}{*}{ UpDown } & Up & 0,000 & 100,000 & 0,000 \\
\hline & & Down & 0,000 & 100,000 & 0,000 \\
\hline & & UpDown & 0,000 & 100,000 & 0,000 \\
\hline \multirow{9}{*}{ UpDown } & \multirow{3}{*}{ Up } & Up & 0,000 & 0,000 & 100,000 \\
\hline & & Down & 0,000 & 0,000 & 100,000 \\
\hline & & UpDown & 0,000 & 0,000 & 100,000 \\
\hline & \multirow{3}{*}{ Down } & Up & 0,000 & 0,000 & 100,000 \\
\hline & & Down & 0,000 & 100,000 & 0,000 \\
\hline & & UpDown & 0,000 & 0,000 & 100,000 \\
\hline & \multirow{3}{*}{ UpDown } & Up & 0,000 & 0,000 & 100,000 \\
\hline & & Down & 0,000 & 0,000 & 100,000 \\
\hline & & UpDown & 0,000 & 0,000 & 100,000 \\
\hline
\end{tabular}

Fig. 8. CMT of $R(k)$.

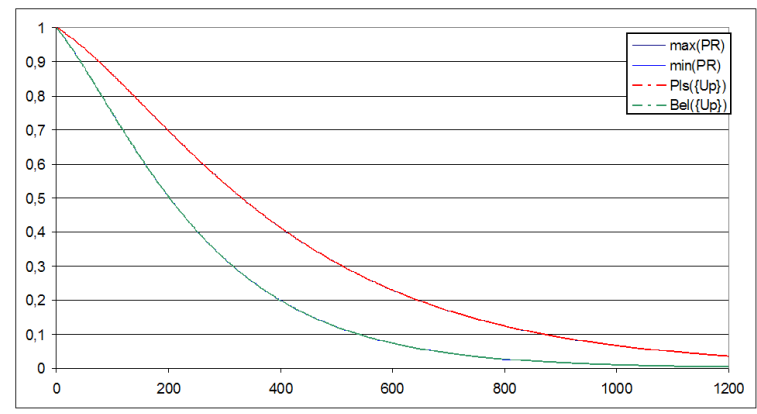

Fig. 9. DEN vs MC simulations.

\section{CONCLUSION AND FURTHER WORK}

In this paper, we have presented Evidential Networks based on the Dempster Shafer theory to model the reliability of systems. A dynamic approach is derived to model the temporal evolution of complex systems with uncertainty. The correspondence between Markov Chain and DEN is presented and applied to the system reliability estimation.

The proposed method seems to be a good solution to model the reliability of complex systems. Indeed, the number of states needed to model a complex system with MC increases exponentially (a state for each combination of elementary states). As the DEN representation is based on the modeling of process entities, the obtained model is more compact and readable than MC. This paper shows that DEN represents a very powerful tool for decision-making aid in maintenance. This modeling method is very interesting when human are taking into account in the model as human actions introduce uncertainty.

\section{REFERENCES}

[1] J. Helton, J. Johnson, and W. Oberkampf, "An exploration of alternative approches to the representation of uncertainty in model predictions," Reliability Engineering \& System Safety, vol. 85, no. 1-3, pp. 39-71, 2004.

[2] G. Klir, "General information theory: aims, results end open problems," Reliability Engineering and System Safety, vol. 85, pp. 21-38, 2004

[3] A. Hoyland and M. Rausand, System reliability theory: models and statistical methods. Wiley, 1994.

[4] C. Simon, P. Weber, and A. Evsukoff, "Bayesian networks inference algorithm to implement dempster shafer theory in reliability analysis," Reliability Engineering and System Safety, vol. 93, no. 7, pp. 950-963, July 2008.

[5] F. Jensen, An Introduction to Bayesian Networks, U. Press, Ed. London: UCL Press, 1996.

[6] S. Padhraic, "Belief networks, hidden markov model, and markov random fields: A unifying view," Pattern recognition letters, vol. 18, pp. 1261-1268, 1997.

[7] C. Boutillier, T. Dean, and S. Hanks, "Decision theoretic planning: structural assumptions and computational leverage," Journal of Artificial Intelligence Research, vol. 11, pp. 1-94, 1999.

[8] CCPS, Offshore reliability data handbook, 4th Edition. SINTEF, 2002.

[9] Exida, Safety Equipment Reliability Handbook, 2nd Edition. Exida, 2005

[10] W. M. Goble and H. Cheddie, Safety Instrumented Systems Verification- Practical Probabilistic Calculations. ISA, 2006.

[11] S. Hauge, H. Langseth, and T. Onshus, Reliability Data for Safety Instrumented Systems, PDS Data Handbook. Sintef, 2006.

[12] IEEE, IEEE guide to the collection and presentation of electrical, electronic, sensing component, and mechanical equipement reliability data for nuclear-power generating station. IEEE-std-500, 1984.

[13] CCPS, Guidelines for process equipement reliability data with data tables. Center for Chemical Process Safety/AIChE, 1991.

[14] T. A. Kletz, HAZOP and HAZAN: Identifying and assessing process industry hazards, 4th edition. Institution of chemical engineers, 1999.

[15] MIL217B, Reliability Prediction of Electronic Equipment. Dept. of Defence, USA, 1974.

[16] A. Villemeur, Reliability, availability, maintainability and safety assessment: methods and techniques. Wiley, 1992.

[17] P. Smets and R. Kennes, "The transferable belief model," Artificial Intelligence, vol. 66, pp. 191-243, 1994.

[18] G. Klir and B. Yuan, Fuzzy Sets and Fuzzy Logic. Theory and Applications., U. S. River, Ed. NJ:Prentice Hall, 1995.

[19] G. Shafer, A Mathematical Theory of Evidence. Princeton University Press, 1976.

[20] M. Valtorta, Y. Kim, and J. Vomlel, "Soft evidential update for probabilistic multiagent systems," International Journal of Approximate Reasoning, vol. 29, pp. 71-106, 2002.

[21] K.-Y. Cai, "System failure engineering and fuzzy methodology: An introductory overview," Fuzzy Sets and Systems, vol. 83, no. 2, pp. 113-133, 1996. [Online]. Available: http://www.sciencedirect.com/science/article/B6V05-3VTK28C1/2/43cc02c0c47c1099a7c4d9a62c238594 